\title{
Excessive Fragmentary Myoclonus: What Do We Know?
}

\section{Jiří Nepožitek, Karel Šonka}

Centre for Sleep and Wake Disorders, Department of Neurology,

First Faculty of Medicine, Charles University and General University Hospital in Prague, Prague, Czech Republic

Received November 6, 2016; Accepted March 9, 2017.

Key words: Fragmentary myoclonus - Excessive fragmentary myoclonus Twitch - Polysomnography - Electromyography - Sleep disorders

\begin{abstract}
Excessive fragmentary myoclonus (EFM) is a polysomnographic finding registered by the surface electromyography (EMG) and characterized as a result of the muscle activity consisting of sudden, isolated, arrhythmic, asynchronous and asymmetric brief twitches. The EMG potentials are defined by the exact criteria in The International Classification of the Sleep Disorders, $3^{\text {rd }}$ edition and they appear with high intensity in all sleep stages. Clinical significance of EFM is unclear. It was observed in combination with other diseases and features such as obstructive and central sleep apnea, narcolepsy, periodic limb movements, insomnia, neurodegenerative disorders and peripheral nerve dysfunction. Relation to such wide range of diseases supports the opinion that EFM is nor a specific sleep disorder nor a specific polysomnographic sign. The option that EFM is a normal variant has also not been ruled out so far.
\end{abstract}

This study was supported by a grant by the Ministry of Health of the Czech Republic, GIGH-16-28914A and a grant by Charles University, GAUK 64216.

Mailing Address: Jiř́ Nepožitek, MD., Department of Neurology, First Faculty of Medicine, Charles University and General University Hospital in Prague, Kateřinská 30, 12000 Prague 2, Czech Republic; Phone: +420 224965 009; e-mail: jiri.nepozitek@lf1.cuni.cz 


\section{Definition and characteristics}

Fragmentary myoclonus (FM) is a polysomnographic phenomenon incidentally discovered in sleep. According to the latest official version of The International Classification of Sleep Disorders, $3^{\text {rd }}$ edition (ICSD-3; American Academy of Sleep Medicine, 2014) by The American Academy of Sleep Medicine (AASM) FM is defined as a result of the muscle activity registered by the surface electromyography (EMG) during polysomnography (PSG). This activity consists of sudden, isolated, arrhythmic, asynchronous and asymmetric brief twitches, jerks or twitch-like movements of muscles or muscle fibers involving various body areas, in particular corners of the mouth, fingers or toes (American Academy of Sleep Medicine, 2014). They do not have to lead to the visible movement every time (Broughton and Tolentino, 1984). The expression "fragmentary" emphasizes the multifocal appearance of the muscle activity. The term fragmentary myoclonus in sleep was first introduced by Broughton and Tolentino in 1984. In ICSD-3 FM is not classified as a nosological entity, it is included among isolated symptoms and normal variants of the sleep related movement disorders (American Academy of Sleep Medicine, 2014). Two forms of FM are being distinguished: the physiologic form and the abnormal (excessive) form.

Physiologic fragmentary myoclonus (PFM) was described by Montagna et al. (1988). This form is called non-excessive fragmentary myoclonus by some authors (Frauscher et al., 2014). The PFM appears as a sporadic phenomenon in rapid eye movement sleep (R) and it is also observed during sleep stage non-REM 1 (N1) with lower rates in wakefulness and stages non-REM 2 (N2) and non-REM 3 (N3 - formerly divided at N3 and non-REM 4) (Montagna et al., 1988).

Excessive fragmentary myoclonus (EFM) is the term first used by Broughton et al. in 1985 in a report of 38 cases. The EFM is described by Broughton et al. (1985) as abnormal amounts of FM potentials, which in contrast to PFM persist throughout all stages of non-REM and REM sleep (Broughton et al., 1985; Mizuma and Sakamoto, 1997; American Academy of Sleep Medicine, 2014).

Criteria determined by the latest official version of ICSD-3 identify the FM as EMG potentials with maximal duration of $150 \mathrm{~ms}$ and amplitude over $50 \mu \mathrm{V}$ rarely rising above $200 \mu \mathrm{V}$ to several hundred $\mu \mathrm{V}$ (American Academy of Sleep Medicine, 2014). The EFM is clinically diagnosed by presence of 5 potentials per minute during at least 20 minutes of recorded non-REM sleep according to the most recent version of the AASM Manual for the Scoring of Sleep and Associated Events 2015 (AASM Manual; Berry et al., 2015).

The EFM during sleep is generally seen in the tibialis anterior muscle surface EMG (Figure 1). It does not tend to appear in bursts unlike phasic REM twitches, which are generally clustered within small groups. The potentials are more universally dispersed throughout a sleep epoch (American Academy of Sleep Medicine, 2014). Patients are usually not aware of the muscle twitches (Lins et al., 1993). EFM is predominantly found in males (Broughton et al., 1985). Although it 


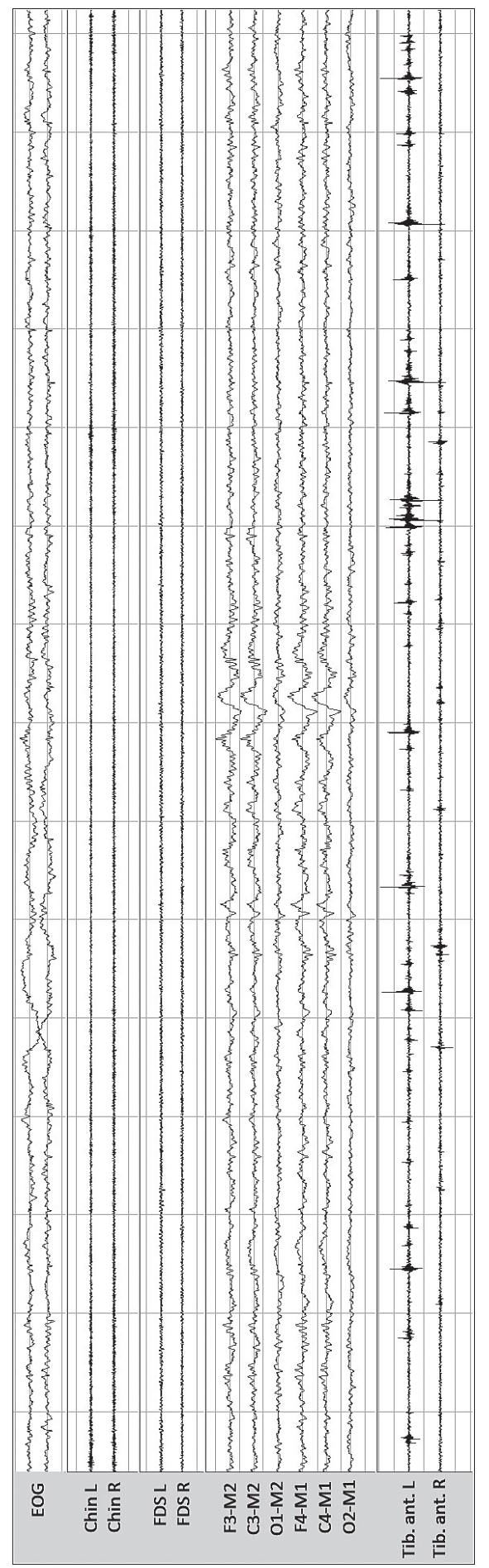

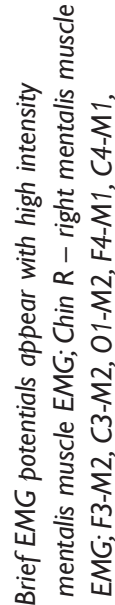

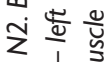

ֻ

जे

ष्षे

出

क्रे

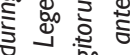

这

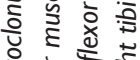

है. ํำ

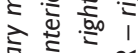

के

ฆ 뜬

हूํํ은

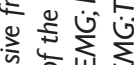

西嵌

ฟे

๖ छ

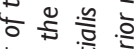

ด bo

这

ํํㄹ

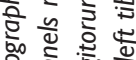

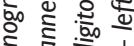

हो ठ ธे ป

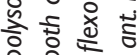

흔

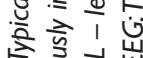

1 ำ

-

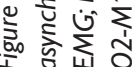


has been described that FM intensity increases with age (Frauscher et al., 2011), it has not yet been confirmed in EFM (Lins et al., 1993).

Occurrence of EFM in wakefulness is not quite clear. In the most recent study Raccagni et al. (2016) report that the EFM potentials extend into wakefulness in a group of 98 patients. FM present in relaxed wakefulness was previously observed by Montagna et al. (1988) and Frauscher et al. (2011). Contradictory to these results is the study with two patients by Vetrugno et al. (2002) who observed EFM purely in sleep.

The results referring about the sleep stage distribution of EFM vary from study to study. It can be summarized that the highest EFM amounts occur in R and the lowest in N3 (Lins et al., 1993; Mizuma and Sakamoto, 1997), while the abnormal intensity in contrast with PFM, is kept. In the recent work by Hoque et al. (2013) the sleep stage distribution was assessed for the different amplitude criteria. For the potentials with lower amplitude criteria ( $\geq 25 \mu \mathrm{V})$ the EFM rates increased from wakefulness to stage N1, 2, 3 and R. With the higher criteria $(40 \mu \mathrm{V}$ and $50 \mu \mathrm{V})$ EFM occurred less often in $\mathrm{N} 3$ than other sleep stages, in which the rate still had an increasing tendency through wakefulness, stages N1, N2 and R (Hoque et al., 2013). Based on the Hoque's results it can be concluded that the EFM intensity increases with N3 and $\mathrm{R}$, while the amplitude of the potentials in non-REM decreases with the fall of muscle tone under the level of the scoring criteria.

The time of night distribution has been studied as well, the number of potentials rise sharply after the initial lower level in the first hour of sleep and remains unchanged across the rest of the night (Lins et al., 1993).

Only one study refers about the prevalence of EFM so far. Frauscher et al. (2014) reported non-excessive FM in every single subject in the group of 100 healthy subjects, whereas $9 \%$ met the criteria for the excessive form.

\section{Development of terminology and assessment}

Sudden, arrhythmic, brief twitches occurring during sleep in various body muscles in men and animals were observed for the first time by De Lisi in 1932, who called the phenomenon physiological hypnic myoclonus. It probably was fragmentary myoclonus.

In 1969, Dagnino et al. used the term hypnic myoclonus for sudden arrhythmic brief twitches observed in sleep at PSG in 18 patients. No amplitude and duration criteria were used in the study. Part of the observed twitches was most likely FM, according to the actual terminology.

Broughton and Tolentino published a case report in 1984, where they used the term sleep related fragmentary pathological multifocal myoclonus and as mentioned above, one year later introduced the term excessive fragmentary myoclonus (Broughton et al., 1985).

In 1988, Montagna et al. used the same term as De Lisi - physiological hypnic myoclonus. Montagna exerted a quantification method, which has not been followed by other authors. 
In 1993, Lins et al. presented a scoring method for the assessment of EFM intensity, called fragmentary myoclonus index (FMI). Each 30-second scoring epoch was divided into ten 3-second mini-epochs. Then the number of mini-epochs with one or more FM potentials exceeding $50 \mu \mathrm{V}$ was counted. Thus this count led to the number ranging from 0 to 10 for each 30 -second epoch. The count then could be referenced to the hours of night and to the duration of individual sleep stages (Lins et al., 1993).

Vetrugno et al. called the phenomenon excessive fragmentary hypnic myoclonus in the case report in 2002. The quantitative analysis was performed only on the left tibialis anterior muscle EMG channel (Vetrugno et al., 2002).

So far, no quantification criteria have been accepted worldwide.

\section{Clinical significance}

A study by Frausher et al. (2011) found FM in 100\% of 62 patients with a variety of sleep disorders. The EFM can be according to the present findings conceivably considered as an abnormal intensification of the PFM however its clinical consequences are unclear. The option that EFM is a normal variant has also not been ruled out so far.

The phenomenon was first noted in the 42-year-old patient suffering from the excessive daytime sleepiness and sleep fragmentation, which was not explained by other sleep disturbances (Broughton and Tolentino, 1984). The EFM has also been described in combination with other sleep disorders such as obstructive sleep apnea, primary central sleep apnea, sleep related hypoxemic and hypoventilation syndromes (Broughton et al., 1985), periodic limb movements disorder (Broughton et al., 1985), narcolepsy (Broughton et al., 1985) and various causes of insomnia (Broughton et al., 1985). In patients with the sleep apnea, the EFM intensifies during periods of increased hypoxemia (American Academy of Sleep Medicine, 2014).

Due to the occurrence of EFM in relation to such sleep comorbidities, the specificity and the causality of EFM as a sole disorder is questionable (American Academy of Sleep Medicine, 2014). A very recent work by Raccagni et al. (2016) confirmed the wide range of coexistent sleep disorders associated with EFM and the findings support the opinion that EFM is not a specific sleep disorder.

A few works have reported the occurrence of EFM in some neurodegenerative diseases. The EFM was observed in Parkinson's disease (Sobreira-Neto et al., 2015), multiple system atrophy (Vetrugno et al., 2007), amyotrophic lateral sclerosis (Sonka et al., 2004), Machado-Joseph disease (dos Santos et al., 2014), NiemannPick disease type C (Vankova et al., 2003) and mitochondrial encephalopathy (Pincherle et al., 2006).

The EFM must be taken into account in differential diagnosis of the following sleep related movement disorders and polysomnographic features: RLS, periodic limb movements in sleep, epileptic myoclonus, sleep related leg cramps, hypnagogic foot tremor, alternating leg muscle activation, and propriospinal myoclonus. 
The RLS are associated with a nocturnal or evening urge to move the legs, uncomfortable and unpleasant sensations in the legs, worsening during periods of rest or inactivity and leading to the limb movements associated with relieving sensations. None of these symptoms is present in EFM (American Academy of Sleep Medicine, 2014).

The periodic limb movements in sleep have in contrast with EFM periodic character. The duration of each movement is longer ( 0.5 to 10 seconds) than FM potentials (American Academy of Sleep Medicine, 2014).

Epileptic myoclonus as an expression of epilepsy in sleep is always associated with an EEG discharge (Lins et al., 1993).

Sleep related leg cramps are sudden and intense involuntary contractions of muscles occurring during the time in bed, unlike EFM lasting much longer (for a few seconds up to several minutes), associated with painful sensations, muscle spasm and hardness (American Academy of Sleep Medicine, 2014).

Hypnagogic foot tremor is rhythmic movement of the feet or toes that occurs at the transition between wake and sleep or during stages N1 and N2 (American Academy of Sleep Medicine, 2014). EFM lacks the rhythmic character and predominant occurrence in mentioned sleep stages.

Alternating leg muscle activation consists of brief repeated activation of the anterior tibialis muscle in one leg in alternation with similar activation in the other leg (American Academy of Sleep Medicine, 2014), they appear in sequences and may recur periodically (Merlino and Gigli, 2012), while EFM lacks the alternating pattern, rhythmicity and periodic occurrence in sequences.

Propriospinal myoclonus consists of sudden jerks, mainly of the abdomen, trunk, and neck. These myoclonic jerks of propriospinal myoclonus irregularly recur when alpha activity is present on the EEG and they disappear with EEG desynchronization and the occurrence of sleep spindles and $\mathrm{K}$-complexes. Myoclonic jerks of propriospinal myoclonus are absent throughout sleep, even if these movements might reappear during intrasleep wakefulness, while EFM is present throughout sleep. The propriospinal myoclonus shows different EMG characteristics than EFM, myoclonic jerks last from 100 to 300 ms (Merlino and Gigli, 2012).

\section{Neurophysiology}

Until 2002 no other neurophysiological investigations of FM except for PSG had been reported. In 2002 Vetrugno et al. performed concentric needle EMG, motor and sensory nerve conduction velocity studies and EEG back-averaging studies. They did not find any neuromuscular abnormalities which could indicate a disorder of integrity of the peripheral nervous system, and they also demonstrated that no cortical EEG potentials preceded the EFM twitches (Vetrugno et al., 2002).

Another work by Gassel et al. (1964) showed the effect of deafferentation on myoclonic twitches in cats. Partial section affecting the dorsal half of the 
lateral funiculus of spinal cord caused a conspicuous reduction in frequency and amplitude of the myoclonic twitches in sleep. The abolition was a global one, not concerning in isolation either extensor or flexor muscle groups, which suggest that neither the pyramidal nor the rubrospinal tract were essential in this regard. The explanation was that reticulospinal tract, as the third important descending system in dorsolateral funiculus, is the effective pathway (Gassel et al., 1964).

Since this excitatory descending volley is physiologically under suppressive control of other structures involved in the active process of sleep, namely rostral and caudal pontine reticular nucleus projecting to gigantocellular reticular nucleus (Chase and Babb, 1973; Chase, 1983; Chase et al., 1986; Takakusaki et al., 1989), the damage or malfunction of these structures and their connections are most likely involved in the etiopathogenesis of EFM. The same structures were identified as the site of origin of the physiological phasic twitches in REM sleep (Kohyama et al., 1994) which implies that both phenomena might have the similar genesis.

The hypothesis that brainstem is the site of origin of EFM is moreover supported by reports of the occurrence of EFM in neurodegenerative diseases associated with the lesions of the brainstem structures (Vankova et al., 2003; Pincherle et al., 2006; Vetrugno et al., 2007; dos Santos et al., 2014; Sobreira-Neto et al., 2015).

Very recently a study by Raccagni et al. (2016) the incidental finding of EFM in 98 patients has shown contradictory results to Vetrugno's conclusions. Fifty percent of the patients in this study presented electrophysiological abnormalities such as polyneuropathy, root lesions and benign fasciculations. Such high prevalence of abnormal neurophysiological findings in patients with EFM points to the possibility that peripheral nerve pathology could be the cause of EFM at least in part of the cases (Raccagni et al., 2016).

Another point of view on the substantiality of EFM was mentioned in the study on amyotrophic lateral sclerosis (Sonka et al., 2004). It was noticed that the characteristics of FM appear to be very similar or identical to the polysomnographic picture of fasciculations. No difference found between particular sleep stages is a feature that resembles the EFM. The fact that hypnic myoclonus displays the characteristics of fasciculation potentials has been noted also by Montagna et al. (1988). A few studies have reported electrophysiologically confirmed benign fasciculation syndrome in the group of EFM patients (Frauscher et al., 2014; Raccagni et al., 2016).

The exact origin of the EFM remains unknown and the results of the existing studies are quite inconsistent so far. Brainstem lesion and peripheral nerve dysfunction considered as the cause of EFM are the two main contradictory hypotheses.

\section{Summary}

The EFM is a challenging phenomenon and there are few existing neurophysiological and clinical studies making a background for the future 
investigations. Its relation to the wide range of sleep disorders supports the opinion that EFM is not a specific sleep disorder and probably not even a specific polysomnographic sign. The option that it is a normal variant has not been disproved yet. Existing results on the pathophysiology of EFM are inconsistent. Observations of EFM in neurodegenerative diseases support the idea that brainstem structures could be involved as the site of EFM origin. Furthermore, it was pointed out that peripheral lesions are associated with EFM as well.

Polysomnographic picture of fasciculations has almost identical characteristics with EFM. There is no doubt that more research has to be done on this topic before the pathophysiological mechanisms and clinical significance of the EFM can be reliably concluded.

\section{References}

American Academy of Sleep Medicine (2014) International Classification of Sleep Disorders, $3^{\text {rd }}$ Ed. American Academy of Sleep Medicine, Darien.

Berry, R. B., Brooks, R., Gamaldo, C. E., Harding, S. M., Lloyd, R. M., Marcus, C. L., Vaughn, B.V. for The American Academy of Sleep Medicine (2015) The AASM Manual for the Scoring of Sleep and Associated Events: Rules, Terminology and Technical Specifications, Version 2.2. American Academy of Sleep Medicine, Darien.

Broughton, R., Tolentino, M. A. (1984) Fragmentary pathological myoclonus in NREM sleep. Electroencephalogr. Clin. Neurophysiol. 57(4), 303-309.

Broughton, R., Tolentino, M. A., Krelina, M. (1985) Excessive fragmentary myoclonus in NREM sleep: a report of 38 cases. Electroencephalogr. Clin. Neurophysiol. 61(2), 123-133.

Chase, M. H. (1983) Synaptic mechanisms and circuitry involved in motoneuron control during sleep. Int. Rev. Neurobiol. 24, 213-258.

Chase, M. H., Babb, M. (1973) Masseteric reflex response to reticular stimulation reverses during active sleep compared with wakefulness or quiet sleep. Brain Res. 59, 421-426.

Chase, M. H., Morales, F. R., Boxer, P. A., Fung, S. J., Soja, P. J. (1986) Effect of stimulation of the nucleus reticularis gigantocellularis on the membrane potential of cat lumbar motoneurons during sleep and wakefulness. Brain Res. 386(1-2), 237-244.

Dagnino, N., Loeb, C., Massazza, G., Sacco, G. (1969) Hypnic physiological myoclonias in man:An EEG-EMG study in normal and neurological patients. Eur. Neurol. 2(1), 47-58.

De Lisi, L. (1932) Su di un fenomeno motorio costante del sonno normale: Le mioclonie ipniche fisiologiche. Riv. Patol. Nerv. Ment. 39, 481-496.

dos Santos, D. F., Pedroso, J. L., Braga-Neto, P., Silva, G. M., de Carvalho, L. B., Prado, L. B., Prado, G. F. (2014) Excessive fragmentary myoclonus in Machado-Joseph disease. Sleep Med. 15(3), 355-358.

Frauscher, B., Kunz, A., Brandauer, E., Ulmer, H., Poewe, W., Hogl, B. (2011) Fragmentary myoclonus in sleep revisited: A polysomnographic study in 62 patients. Sleep Med. 12(4), 410-415.

Frauscher, B., Gabelia, D., Mitterling, T., Biermayr, M., Bregler, D., Ehrmann, L., Hogl, B. (2014) Motor events during healthy sleep: A quantitative polysomnographic study. Sleep 37(4), 763-773, 773A-773B.

Gassel, M. M., Marchiafava, P. L., Pompeiano, O. (1964) Phasic changes in muscular activity during desynchronized sleep in unrestrained cats. An analysis of the pattern and organization of myoclonic twitches. Arch. Ital. Biol. 102, 449-470.

Hoque, R., McCarty, D. E., Chesson, A. L. Jr. (2013) Manual quantitative assessment of amplitude and sleep stage distribution of excessive fragmentary myoclonus. J. Clin. Sleep Med. 9(1), 39-45.

Nepožitek J.; Šonka K. 
Kohyama, J., Shimohira, M., Iwakawa, Y. (1994) Brainstem control of phasic muscle activity during REM sleep: a review and hypothesis. Brain Dev. 16(2), 81-91.

Lins, O., Castonguay, M., Dunham, W., Nevsimalova, S., Broughton, R. (1993) Excessive fragmentary myoclonus: Time of night and sleep stage distributions. Can. J. Neurol. Sci. 20(2), 142-146.

Merlino, G., Gigli, G. L. (2012) Sleep-related movement disorders. Neurol. Sci. 33(3), 491-513.

Mizuma, H., Sakamoto, T. (1997) Excessive twitch movements in rapid eye movement sleep with daytime sleepiness. Psychiatry Clin. Neurosci. 51(6), 393-396.

Montagna, P., Liguori, R., Zucconi, M., Sforza, E., Lugaresi, A., Cirignotta, F., Lugaresi, E. (1988) Physiological hypnic myoclonus. Electroencephalogr. Clin. Neurophysiol. 70(2), 172-176.

Pincherle, A., Mantoani, L., Villani, F., Confalonieri, P., Erbetta, A. (2006) Excessive fragmentary hypnic myoclonus in a patient affected by a mitochondrial encephalomyopathy. Sleep Med. 7(8), 663.

Raccagni, C., Löscher,W. N., Stefani, A., Wanschitz, J., Kraemer, L., Heidbreder, A., Högl, B. (2016) Peripheral nerve function in patients with excessive fragmentary myoclonus during sleep. Sleep Med. 22, 61-64.

Sobreira-Neto, M. A., Pena-Pereira, M. A., Sobreira, E. S., Chagas, M. H., Tumas, V., Fernandes, R. M., Eckeli, A. L. (2015) Excessive fragmentary myoclonus in patients with Parkinson's disease: Prevalence and clinicopolysomnographic profile. Sleep Breath. 19(3), 997-1002.

Sonka, K., Fiksa, J., Horvath, E., Kemlink, D., Sussova, J., Bohm, J., Nevsimalova, S. (2004) Sleep and fasciculations in amyotrophic lateral sclerosis. Somnologie 8, 25-30.

Takakusaki, K., Ohta, Y., Mori, S. (1989) Single medullary reticulospinal neurons exert postsynaptic inhibitory effects via inhibitory interneurons upon alpha-motoneurons innervating cat hindlimb muscles. Exp. Brain Res. 74(1), 11-23.

Vankova, J., Stepanova, I., Jech, R., Elleder, M., Ling, L., Mignot, E., Nevsimalova, S. (2003) Sleep disturbances and hypocretin deficiency in Niemann-Pick disease type C. Sleep 26(4), 427-430.

Vetrugno, R., Plazzi, G., Provini, F., Liguori, R., Lugaresi, E., Montagna, P. (2002) Excessive fragmentary hypnic myoclonus: Clinical and neurophysiological findings. Sleep Med. 3(1), 73-76.

Vetrugno, R., Liguori, R., Cortelli, P., Plazzi, G., Vicini, C., Campanini, A., Montagna, P. (2007) Sleep-related stridor due to dystonic vocal cord motion and neurogenic tachypnea/tachycardia in multiple system atrophy. Mov. Disord. 22(5), 673-678. 